Research article

Open Access

\title{
Antiarrhythmic Activities of Some Newly Synthesized Tricyclic and Tetracyclic Thienopyridine Derivatives
}

\author{
Naglaa A. Abdel-Hafez * ${ }^{1}$, Ashraf, M. Mohamed ${ }^{1}$, \\ Abd El-Galil E. AMr ${ }^{1}$, Mohamed M. ABDALLA ${ }^{2}$ \\ ${ }^{1}$ Applied Organic Chemistry Department, National Research Centre, Dokki, Cairo Egypt \\ ${ }^{2}$ Research Units, Hi-Care Pharmaceutical Co., Cairo, Egypt \\ * Corresponding author. E-mail: rahma14_4@yahoo.com (N. A. Abdel-Hafez) \\ Sci Pharm. 2009; 77: 539-553 \\ doi:10.3797/scipharm.0905-06 \\ Published: $\quad$ August $5^{\text {th }} 2009$ \\ Accepted: $\quad$ August $4^{\text {th }} 2009$ \\ Received: $\quad$ May $11^{\text {th }} 2009$ \\ This article is available from: http://dx.doi.org/10.3797/scipharm.0905-06 \\ (C) Abdel-Hafez et al.; licensee Österreichische Apotheker-Verlagsgesellschaft m. b. H., Vienna, Austria. \\ This is an Open Access article distributed under the terms of the Creative Commons Attribution License \\ (http://creativecommons.org/licenses/by/3.0/), which permits unrestricted use, distribution, and reproduction \\ in any medium, provided the original work is properly cited.
}

\begin{abstract}
3,5-Bis(4-chlorobenzylidene)-1-ethylpiperidin-4-one (1b) was condensed with malononitrile or cyanothioacetamide to yield pyranopyridine 2 and thiopyridopyridine $\mathbf{3 b}$, respectively. Treatment of compound $\mathbf{3 b}$ with methyl iodide or ethyl chloroacetate in the presence of a base catalyst gave the corresponding compounds $\mathbf{4}$ and $\mathbf{5}$. Compound $\mathbf{3 b}$ was reacted with 2 -chloro- $N$-arylacetamide derivatives to yield compounds $7 \mathbf{a}, \mathbf{b}$, which were reacted with benzoyl chloride or sodium nitrite to give the corresponding tetracyclic compounds $\mathbf{8} \mathbf{a}, \mathbf{b}$ and $\mathbf{9 a}, \mathbf{b}$, respectively. Compound $\mathbf{2}$ was treated with acetic anhydride or formic acid to yield the corresponding $N$-acetylpyranopyridine 10 and pyranopyrimidine 11. Treatment of compound 2 with triethyl ortho-formate gave compound $\mathbf{1 2}$, which was cyclized with hydrazine hydrate to give $\mathrm{N}$-aminopyrimidine 13 . Some of the synthesized compounds showed high antiarrhythmic activities comparable with Procaine amide and Lidocaine as positive controls.
\end{abstract}

\section{Keywords}

Pyridinethione - Thienopyridine - Thienopyrimidine - Thienotriazine Pyranopyrimidine • Antiarrhythmic agents 


\section{Introduction}

The search for new heterocyclic compounds and novel methods for their synthesis is a major topic in contemporary organic synthesis. As part of our program in this area, we have synthesized some pyridine and thiazolopyrimidine derivatives of biological interest as anticancer activity [1-5]. On the other hand, thienopyrimidine and thioxopyrimidine derivatives have promising biological $[6,7]$ and inhibitor of VEGFR-2 kinase activity $[8,9]$. Recently, some new pyridine, pyrimidine and their derivatives have been synthesized and used as analgesic, anticonvulsant and anti-parkinsonian agents [10-15]. In addition, Atheral et al [16] have synthesized a novel series of thieno[2,3-d]pyrimidines which can be used in combating fungi in plant and inhibited the growth of cancer cells $[17,18]$. In continuation of our previous work we synthesized some new tricyclic and tetracyclic heterocyclic compounds containing tetrahydropyridopyridine nucleus and tested their antiarrhythmic activities.

\section{Results and Discussion}

3,5-Bis(benzylidene)-1-ethylpiperidin-4-ones 1a-c and pyranopyridine derivative 2 were used as starting materials and prepared according to the published method [3]. Compounds 1a-c were reacted with cyanothioacetamide in refluxing ethanol containing drops of $\mathrm{Et}_{3} \mathrm{~N}$ to give pyridinethione derivatives $3 \mathrm{a}-\mathrm{c}$, which were reacted with methyl iodide in sodium ethoxide solution with stirring at room temperature to yield the methylmercaptopyridine derivative 4. Similarly, compound $\mathbf{3 b}$ was reacted with ethyl chloroacetate in refluxing ethanol in the presence of drops of $\mathrm{Et}_{3} \mathrm{~N}$ as a catalyst to give the thienopyridine derivative 5 (Sch. 1).

Compound 3 b was reacted with 2 -chloro- $N$-arylacetamide derivatives namely, 2-chloro- $N$ ( $p$-tolyl)acetamide or 2-chloro- $N$-(4-fluorophenyl)acetamide in refluxing ethanol containing drops of $\mathrm{Et}_{3} \mathrm{~N}$ to yield 2-(N-aryl)-carboxamidomethylthiopyridine derivatives $\mathbf{6 a}, \mathbf{b}$, which were cyclized by boiling in sodium ethoxide solution to afford the corresponding 2-(N-aryl)carboxamidomethyl-thienopyridine derivatives $\mathbf{7 a , b}$. The latter compound $\mathbf{7 a , b}$ were also obtained directly from the reaction of compound $\mathbf{3 b}$ with 2-chloro-N-arylacetamide derivatives in refluxing sodium ethoxide solution. Fusion of compounds $\mathbf{7 a , b}$ with an excess of benzoyl chloride gave pyridothieno[3,2-d]pyrimidin-4-one derivatives 8a,b. Moreover, treatment of compounds $\mathbf{7 a}, \mathbf{b}$ in $\mathrm{HCl} / \mathrm{AcOH}$ mixture with sodium nitrite solution at $0{ }^{\circ} \mathrm{C}$ affording thieno[3,2-d][1,2,3]triazin-4-one derivatives $9 \mathbf{a}, \mathbf{b}$ (Sch. 2).

Compound 2 was reacted with acetic anhydride or formic acid to yield $\mathrm{N}$-acetylpyranopyridine 10 and pyranopyrimidine derivative 11, respectively. Compound 2 was condensed with triethyl ortho-formate in refluxing acetic anhydride to give the corresponding ethoxymethylidenamino derivative 12, which was cyclized with hydrazine hydrate in ethanol at room temperature with stirring to give $\mathrm{N}$-aminopyrimidine derivative 13 (Sch. 3). 
<smiles>[X]c1ccc(C=C2CN(CC)CC(=Cc3ccccc3)C2=O)cc1</smiles>

$1 \mathrm{a}-\mathrm{c}$<smiles>CC(C)(N)CC#N</smiles><smiles>[X]c1cccc(/C=C2\CN(CC)Cc3c2[nH]c(=S)c(C#N)c3-c2ccccc2)c1</smiles>

3a-c

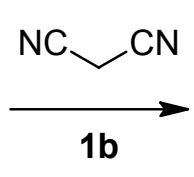

2<smiles>CCN1CC(=Cc2ccc(Cl)cc2)C2=C(C1)C(c1ccc(Cl)cc1)C(C#N)=C(N)O2</smiles>

a: $X=H$

b: $X=4-\mathrm{Cl}$

c: $\mathrm{X}=3,4,5-\left(\mathrm{OCH}_{3}\right)_{3}$<smiles>CCOC(=O)c1sc2nc3c(c(-c4ccc(Cl)cc4)c2c1N)CN(CC)C/C3=C\c1ccc(Cl)cc1</smiles>

5<smiles>CCOC(=O)CCl</smiles><smiles></smiles><smiles>CCN1C/C(=C\c2ccc(Cl)cc2)c2nc(S(C)(=O)=O)c(C#N)c(-c3ccc(Cl)cc3)c2C1</smiles>

4

Sch. 1. 
<smiles>CCN1CC(=Cc2ccc(Cl)cc2)c2[nH]c(=S)c(C#N)c(-c3ccc(Cl)cc3)c2C1</smiles>

3b<smiles>CCCC(C)(Cl)COC(=O)NCCOCC</smiles><smiles>CCN1C/C(=C\c2ccc(Cl)cc2)c2nc(SCC(=O)N[Ga])c(C#N)c(-c3ccc(Cl)cc3)c2C1</smiles>

$6 a, b$

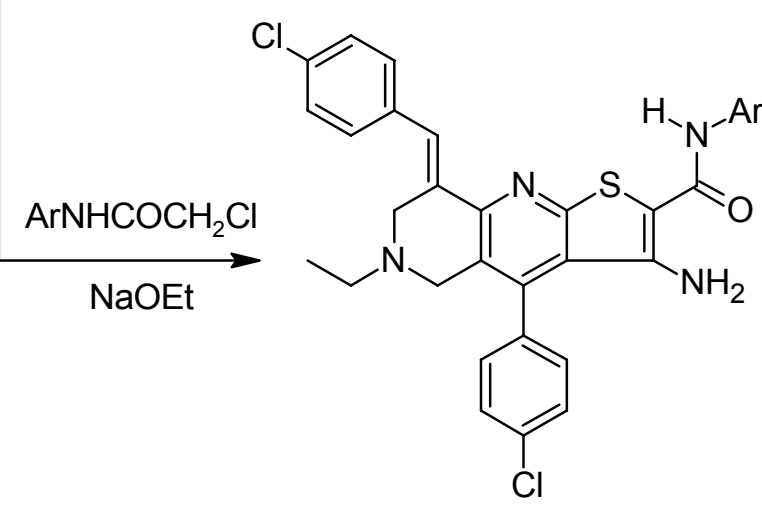

NaOEt

7a,b

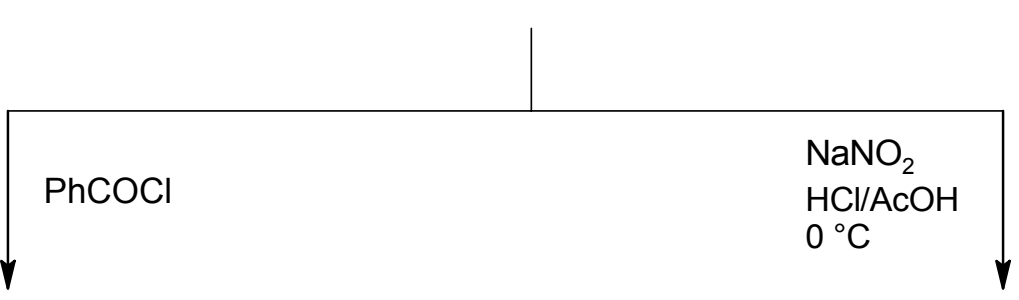

$\mathrm{Cl}$<smiles>CCN1CC(=Cc2ccc(C)cc2)c2nc3sc4c(=O)n(C(C)Br)c(-c5ccccc5)nc4c3c(-c3ccc(Cl)cc3)c2C1</smiles>

$\mathrm{Cl}$<smiles>CCN1C/C(=C\c2ccc(I)cc2)c2nc3sc4c(=O)n(C(C)C)nnc4c3c(-c3ccc(Cl)cc3)c2C1</smiles>

$8 a, b$

a: $\mathrm{Ar}=p-\mathrm{C}_{6} \mathrm{H}_{4} \mathrm{CH}_{3}$

9a,b

b: $\mathrm{Ar}=p-\mathrm{C}_{6} \mathrm{H}_{4} \mathrm{~F}$

Sch. 2. 
$\mathrm{Cl}$<smiles>CCN1CC2=C(OC(NC(C)=O)=C(C#N)C2c2ccc(Cl)cc2)/C(=C/c2ccc(I)cc2)C1</smiles>

10
$\mathrm{Cl}$<smiles>CCN1CC2=C(OC(N)=C(C#N)C2c2ccc(Cl)cc2)/C(=C/c2ccc(F)cc2)C1</smiles>

2<smiles>CCN1CC(=Cc2ccc(Cl)cc2)C2=C(C1)C(c1ccc(Cl)cc1)c1c(nc[nH]c1=O)O2</smiles>

11<smiles>CCOC=NC1=C(C#N)C(c2ccc(Cl)cc2)C2=C(C1)/C(=C/c1ccc(Cl)cc1)CN(CC)C2</smiles>

12
$\mathrm{NH}_{2} \mathrm{NH}_{2} \cdot \mathrm{H}_{2} \mathrm{O}$ $\mathrm{HC}(\mathrm{OEt})_{3}$

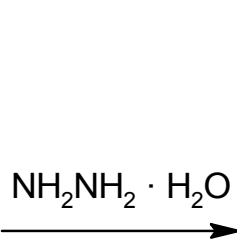

Sch. 3.

\section{Pharmacological Screening}

The newly synthesized compounds were pharmacologically screened for their Antiarrhythmic potency (Table 1).

Procaine amide, $5 \mathrm{mg} / \mathrm{kg}$ iv and lidocaine $5 \mathrm{mg} / \mathrm{kg}$ i.v. led to an increase in $\mathrm{LD}_{100}$ by $65 \%$, which corresponds to a $L_{100}$ of approximately $9 \mu \mathrm{g} / 100 \mathrm{mg}$. 
From Table 1, Compounds 6a, 3c, 12 and 13 displayed nearly equal anti-arrhythmic activities as procaine amide and lidocaine. Compounds $\mathbf{1 b}, \mathbf{4}, \mathbf{5}, \mathbf{7 b}, \mathbf{8 a}, \mathbf{8 b}, \mathbf{9 a}$, and $\mathbf{9 b}$ are more active than procaine amide and lidocaine, they are arranged in descending manner. Also, 2, 3a, 3b, 6b, 7a, 10 and 11 showed anti-arrhythmic activities less than procaine and lidocaine, they are arranged in descending manner.

Tab. 1. Anti-arrhythmic activities of the newly synthesized compounds

\begin{tabular}{|c|c|}
\hline $\begin{array}{l}\text { Compound in } \\
(5 \mathrm{mg} / \mathrm{kg})\end{array}$ & $\begin{array}{l}\text { Percentage } \\
\text { Increase in } L D_{100}\end{array}$ \\
\hline 1 & $76 \pm 0.08$ \\
\hline $2 b$ & $54 \pm 0.05$ \\
\hline $3 a$ & $48 \pm 0.05$ \\
\hline $3 b$ & $41 \pm 0.05$ \\
\hline $3 c$ & $67 \pm 0.07$ \\
\hline 4 & $79 \pm 0.07$ \\
\hline 5 & $77 \pm 0.09$ \\
\hline $6 a$ & $65 \pm 0.06$ \\
\hline $6 b$ & $45 \pm 0.05$ \\
\hline $7 a$ & $47 \pm 0.04$ \\
\hline $7 b$ & $77 \pm 0.08$ \\
\hline $8 a$ & $75 \pm 0.08$ \\
\hline $8 b$ & $86 \pm 0.09$ \\
\hline $9 a$ & $72 \pm 0.07$ \\
\hline $9 b$ & $78 \pm 0.10$ \\
\hline 10 & $37 \pm 0.05$ \\
\hline 11 & $55 \pm 0.06$ \\
\hline 12 & $69 \pm 0.07$ \\
\hline 13 & $68 \pm 0.07$ \\
\hline
\end{tabular}

\section{Structural Activity Relationship (SAR)}

- The naphthyridine moiety and a high degree of aromaticity is essential for antiarrhythmic activity.

- Hetero-aromaticity increases the anti-arrhythmic activity

- Fused ring systems give anti-arrhythmic activity but to a lower extent.

\section{Experimental}

All melting points were taken on a Electrothermal IA 9000 series digital melting point apparatus. Elemental analytical data (in accordance with the calculated values) were obtained from the microanalytical unit, Cairo University, Cairo, Egypt. The IR spectra (KBr) were recorded on a Pye Unicam SP-1000 spectrophotometer. The ${ }^{1} \mathrm{H} N M R$ and ${ }^{13} \mathrm{C}$ NMR spectra were recorded at $270 \mathrm{MHz}$ and $64.5 \mathrm{MHz}$, respectively, on a Varian EM-360 
Spectrometer using TMS as an internal standard. The mass spectra were performed using a VC 2AB-3F spectrometer $(70 \mathrm{eV})$. All reactions were followed by TLC (silica gel, aluminium sheets $60 \mathrm{~F}_{254}$, Merck).

\section{(8E)-4-aryl-8-(arylmethylidene)-6-ethyl-2-thioxo-1,2,5,6,7,8-hexahydro- 1,6-naphthyridine-3-carbonitriles (3a-c)}

A mixture of compound $1 \mathrm{a}-\mathrm{c}(0.01 \mathrm{~mole})$, cyanothioacetamide $(0.10 \mathrm{~g}, 0.01 \mathrm{~mole})$ and few drops of $\mathrm{Et}_{3} \mathrm{~N}$ in ethanol $(100 \mathrm{ml})$ was refluxed for $6 \mathrm{~h}$. The reaction mixture was poured onto cold water and acidified with $\mathrm{HCl}$ to $\mathrm{pH} 3$, the orange solid formed was filtered off, washed with water, dried and recrystallized to give $\mathbf{3 a - c .}$

(8E)-8-Benzylidene-6-ethyl-4-phenyl-2-thioxo-1,2,5,6,7,8-hexahydro1,6-naphthyridine-3-carbonitrile (3a)

Yield (55\%), M.p. $205^{\circ} \mathrm{C}$ (Ethanol); IR (KBr) v = $3345(\mathrm{NH}), 2212(\mathrm{CN}) \mathrm{cm}^{-1} ; \mathrm{MS} \mathrm{m} / \mathrm{z}(\%)$ : $383\left[\mathrm{M}^{+}\right]$base peak (100) corresponding to the molecular formula $\mathrm{C}_{24} \mathrm{H}_{21} \mathrm{~N}_{3} \mathrm{~S}, 354$ (79). Analysis for $\mathrm{C}_{24} \mathrm{H}_{21} \mathrm{~N}_{3} \mathrm{~S}$ (383.51): Calc. $\mathrm{C}, 75.16 ; \mathrm{H}, 5.52 ; \mathrm{N}, 10.96$; $\mathrm{S}, 8.36$, Found $\mathrm{C}$, 75.12; H, 5.56; N, 11.02; S, 8.26.

\section{(8E)-8-(4-chlorobenzylidene)-4-(4-chlorophenyl)-6-ethyl-2-thioxo-}

\section{1,2,5,6,7,8-hexahydro-1,6-naphthyridine-3-carbonitrile (3b)}

Yield (50\%), M.p. $228{ }^{\circ} \mathrm{C}(\mathrm{EtOH})$; IR $(\mathrm{KBr})$ v $=3320(\mathrm{NH}), 2208(\mathrm{CN}) \mathrm{cm}^{-1}$; ${ }^{1} \mathrm{H}-\mathrm{NMR}$ $\left(\mathrm{CDCl}_{3}\right) \delta=0.9\left(\mathrm{t}, 3 \mathrm{H}, \mathrm{CH}_{3}\right), 2.40\left(\mathrm{q}, 2 \mathrm{H}, \mathrm{CH}_{2}\right), 3.33,3.42\left(2 \mathrm{~s}, 4 \mathrm{H}, 2 \mathrm{CH}_{2}\right), 7.30-7.80(\mathrm{~m}$, $9 \mathrm{H}, \mathrm{ArH}+$ benzylic proton), $7.95\left(\mathrm{~s}, 1 \mathrm{H}, \mathrm{NH}\right.$, exchangeable with $\left.\mathrm{D}_{2} \mathrm{O}\right) ;{ }^{13} \mathrm{C}-\mathrm{NMR}\left(\mathrm{CDCl}_{3}\right) \delta$ $=13.35\left(\mathrm{CH}_{3}\right), 50.55,51.45,58.90\left(3 \mathrm{CH}_{2}\right), 125.30,135.25(\mathrm{C}=\mathrm{C}), 103.25,114.55$, 145.50, 153.62 (Pyridine-C),117.95 (CN), 125.50, 126.75, 128.55, 129.15, 128.30, 129.10, 132.57, 133.50 (Ph-C), $165.25(\mathrm{C}=\mathrm{S}) ; \mathrm{MS} \mathrm{m} / \mathrm{z}(\%): 451\left[\mathrm{M}^{+}\right]$base peak (100) corresponding to the molecular formula $\mathrm{C}_{24} \mathrm{H}_{19} \mathrm{Cl}_{2} \mathrm{~N}_{3} \mathrm{~S}, 453$ [M+2] (80), 455 [M+4] (30), 326 (79). Analysis for $\mathrm{C}_{24} \mathrm{H}_{19} \mathrm{Cl}_{2} \mathrm{~N}_{3} \mathrm{~S}$ (452.40): Calc. C, 63.72; $\mathrm{H}, 4.23 ; \mathrm{Cl}, 15.67 ; \mathrm{N}, 9.29 ; \mathrm{S}$, 7.09, Found C, 63.69; H, 4.20; Cl, 15.63; N, 9.32; S, 7.12.

\section{(8E)-6-Ethyl-2-thioxo-4-(3,4,5-trimethoxyphenyl)-8-(3,4,5-trimethoxybenzylidene)-} 1,2,5,6,7,8-hexahydro-1,6-naphthyridine-3-carbonitrile (3c)

Yield (70\%), M.p. $255^{\circ} \mathrm{C}$ (Ethanol); IR (KBr) v = $3358(\mathrm{NH}), 2220(\mathrm{CN}) \mathrm{cm}^{-1}$; MS m/z (\%): $563\left[\mathrm{M}^{+}\right]$base peak (100) corresponding to the molecular formula $\mathrm{C}_{30} \mathrm{H}_{33} \mathrm{~N}_{3} \mathrm{SO}_{6}, 543(80)$, 394 (75). Analysis for $\mathrm{C}_{30} \mathrm{H}_{33} \mathrm{~N}_{3} \mathrm{SO}_{6}$ (563.66): Calc. C, 63.92; $\mathrm{H}, 5.90 ; \mathrm{N}, 7.45 ; \mathrm{S}, 5.69$, Found C, 63.88; H, 5.94; N, 7.48; S, 5.73

(8E)-8-(4-Chlorobenzylidene)-4-(4-chlorophenyl)-6-ethyl-2-(methylsulfanyl)5,6,7,8-tetrahydro-1,6-naphthyridine-3-carbonitrile (4)

A mixture of compound $3 \mathrm{~b}$ ( $0.45 \mathrm{~g}, 0.001 \mathrm{~mole})$ and methyl iodide $10 \%$ excess was suspended in sodium ethoxide solution (10 mg sodium metal in $50 \mathrm{ml}$ ethanol) and stirred for $1 \mathrm{~h}$. at room temperature. The reaction mixture was poured onto cold water, the obtained solid was filtered off, dried and recrystallized to afford 4. Yield (50\%), M.p. $150{ }^{\circ} \mathrm{C}$ (Ethanol) IR $(\mathrm{KBr}) \mathrm{v}=2217(\mathrm{CN}) \mathrm{cm}^{-1} ;{ }^{1} \mathrm{H}-\mathrm{NMR}\left(\mathrm{CDCl}_{3}\right) \delta: 1.10\left(\mathrm{t}, 3 \mathrm{H}, \mathrm{CH}_{3}\right), 2.37(\mathrm{q}, 2 \mathrm{H}$, $\left.\mathrm{CH}_{2}\right), 2.70\left(\mathrm{~s}, 3 \mathrm{H}, \mathrm{CH}_{3}\right), 3.20-3.35\left(\mathrm{~m}, 4 \mathrm{H}, 2 \mathrm{CH}_{2}\right) 7.20-7.95(\mathrm{~m}, 9 \mathrm{H}, \mathrm{ArH}+$ benzylic proton); $\mathrm{MS} \mathrm{m} / \mathrm{z}(\%): 466\left[\mathrm{M}^{+}\right]$(85) corresponding to the molecular formula $\mathrm{C}_{25} \mathrm{H}_{21} \mathrm{Cl}_{2} \mathrm{~N}_{3} \mathrm{~S}$, 
468 [M+2] (base peak 100), 337 (68). Analysis for $\mathrm{C}_{25} \mathrm{H}_{21} \mathrm{Cl}_{2} \mathrm{~N}_{3} \mathrm{~S}$ (466.43): Calc. C, 64.38; H, 4.54; Cl, 15.20; N, 9.01; S, 6.87, Found C, 64.32; H, 4.58; Cl, 15.15; N, 9.06; S, 6.83.

\section{Ethyl (8E)-3-amino-4-(4-chlorophenyl)-8-(4-chlorobenzylidene)-6-ethyl- 5,6,7,8-tetrahydrothieno[2,3-b][1,6]naphthyridine-2-carboxylate (5)}

To a mixture of compound $3 \mathbf{b}(0.45 \mathrm{~g}, 0.001$ mole) and ethyl chloroacetate $(0.14 \mathrm{~g}, 0.001$ mole) in ethanol $(50 \mathrm{ml})$, few drops of $\mathrm{Et}_{3} \mathrm{~N}$ were added then the reaction mixture was refluxed for $4 \mathrm{~h}$. The solid formed was filtered off, dried and recrystallized to give $\mathbf{5}$. Yield (35\%), M.p. $238{ }^{\circ} \mathrm{C}$ (Ethanol), IR (KBr) v = 3410, $3430\left(\mathrm{NH}_{2}\right), 1738$ (CO) $\mathrm{cm}^{-1}$; ${ }^{1} \mathrm{H}-\mathrm{NMR}$ (DMSO-d 6 ) ס: 1.05, $1.10\left(2 \mathrm{t}, 6 \mathrm{H}, 2 \mathrm{CH}_{3}\right), 2.46$ (q, 2H, $\left.\mathrm{CH}_{2}\right), 3.29-3.35\left(\mathrm{~m}, 4 \mathrm{H}, 2 \mathrm{CH}_{2}\right), 4.05$ (q, 2H, $\mathrm{CH}_{2}$ ), 4.55 (s, 2H, NH $\mathrm{N}_{2}$ ), 7.50-7.90 (m, 9H, ArH + benzylic proton); MS m/z (\%): $538\left[\mathrm{M}^{+}\right]$base peak (100) corresponding to the molecular formula $\mathrm{C}_{28} \mathrm{H}_{25} \mathrm{Cl}_{2} \mathrm{~N}_{3} \mathrm{SO}_{2}, 412$ (86). Analysis for $\mathrm{C}_{28} \mathrm{H}_{25} \mathrm{Cl}_{2} \mathrm{~N}_{3} \mathrm{SO}_{2}$ (538.49): Calc. C, 62.45; $\mathrm{H}, 4.68 ; \mathrm{Cl}, 13.17 ; \mathrm{N}, 7.80 ; \mathrm{S}$, 5.95, Found $\mathrm{C}, 62.41 ; \mathrm{H}, 4.62 ; \mathrm{Cl}, 13.13 ; \mathrm{N}, 7.83 ; \mathrm{S}, 5.91$.

\section{2-(\{(8E)-4-(4-Chlorophenyl)-8-(4-chlorobenzyliden)-3-cyano-6-ethyl- 5,6,7,8-tetrahydro-1,6-naphthyridin-2-yl\} sulfanyl)-N-arylacetamides (6a,b)}

\section{Method A:}

To a mixture of compound $\mathbf{3 b}(0.45 \mathrm{~g}, 0.001$ mole) and anhydrous sodium acetate $(2 \mathrm{~g})$ in ethanol $(50 \mathrm{ml}), 2$-chloro- $N$-arylacetamide, namely, 2-chloro- $N$-( $p$-tolyl)acetamide or 2 -chloro- $N$-(4-fluorophenyl)acetamide $(0.001$ mole $)$ was added. The reaction mixture was refluxed for $2 \mathrm{~h}$ then cooled and poured onto cold water. The solid formed was filtered off, dried and recrystallized to give $\mathbf{6} \mathbf{a}, \mathbf{b}$ respectively.

\section{Method B:}

To a mixture of compound $3 \mathbf{b}(0.45 \mathrm{~g}, 0.001 \mathrm{~mole})$ and 2-chloro- $N$-arylacetamide namely, 2-chloro- $N$ - $(p$-tolyl)acetamide or 2-chloro- $N$-(4-fluorophenyl)acetamide $(0.001$ mole) in ethanol $(50 \mathrm{ml})$, few drops of $\mathrm{Et}_{3} \mathrm{~N}$ were added then the reaction mixture was refluxed for $3 \mathrm{~h}$. The solid formed was filtered off, dried and recrystallized to give $\mathbf{6} \mathbf{a}, \mathbf{b}$ respectevely.

\section{2-(\{(8E)-4-(4-Chlorophenyl)-8-(4-chlorobenzylidene)-3-cyano-6-ethyl-}

\section{5,6,7,8-tetrahydro-1,6-naphthyridin-2-yl\} sulfanyl)-N-(4-methylphenyl)acetamide (6a)}

Yield (55\%) [A], (75\%) [B], M.p. $218{ }^{\circ} \mathrm{C}$ (Dioxan), IR (KBr) v = $3294(\mathrm{NH}), 2215(\mathrm{CN})$ and $1662(\mathrm{CO}) \mathrm{cm}^{-1} ;{ }^{1} \mathrm{H}-\mathrm{NMR}\left(\mathrm{CDCl}_{3}\right) \delta: 0.98\left(\mathrm{t}, 3 \mathrm{H}, \mathrm{CH}_{3}\right), 2.27\left(\mathrm{~s}, 3 \mathrm{H}, \mathrm{CH}_{3}\right), 2.50(\mathrm{q}, 2 \mathrm{H}$, $\left.\mathrm{CH}_{2}\right), 3.40,3.70\left(2 \mathrm{~d}, 4 \mathrm{H}, 2 \mathrm{CH}_{2}\right), 4.10\left(\mathrm{~s}, 2 \mathrm{H}, \mathrm{CH}_{2}\right) 7.10-7.83(\mathrm{~m}, 13 \mathrm{H}$, ArH + bezilic proton), $8.20\left(\mathrm{~s}, 1 \mathrm{H}, \mathrm{NH}\right.$, exchangeable with $\left.\mathrm{D}_{2} \mathrm{O}\right) ; \mathrm{MS} \mathrm{m} / \mathrm{z}(\%)$ : $\left.599 \mathrm{M}^{+}\right]$(55) corresponding to the molecular formula $\mathrm{C}_{33} \mathrm{H}_{28} \mathrm{Cl}_{2} \mathrm{~N}_{4} \mathrm{SO}, 449$ base peak (100). Analysis for $\mathrm{C}_{33} \mathrm{H}_{28} \mathrm{Cl}_{2} \mathrm{~N}_{4} \mathrm{SO}$ (599.58): Calc. C, 66.11; H, 4.71; Cl, 11.83; N, 9.34; S, 5.35, Found C, $66.15 ; \mathrm{H}, 4.76 ; \mathrm{Cl}, 11.86 ; \mathrm{N}, 9.30 ; \mathrm{S}, 5.38$

2-(\{(8E)-4-(4-Chlorophenyl)-8-(4-chlorobenzylidene)-3-cyano-6-ethyl5,6,7,8-tetrahydro-1,6-naphthyridin-2-yl\}sulfanyl)-N-(4-fluorphenyl)acetamide (6b)

Yield (60\%) [A], (78\%) [B], M.p. $205{ }^{\circ} \mathrm{C}$ (Dioxan), IR (KBr) v = $3315(\mathrm{NH}), 2210(\mathrm{CN})$ and $1655(\mathrm{CO}) \mathrm{cm}^{-1}$. ${ }^{1} \mathrm{H}-\mathrm{NMR}\left(\mathrm{CDCl}_{3}\right) \delta: 1.05\left(\mathrm{t}, 3 \mathrm{H}, \mathrm{CH}_{3}\right), 2.35$ (q, 2H, $\left.\mathrm{CH}_{2}\right), 3.35,3.60(2 \mathrm{~d}$, $4 \mathrm{H}, 2 \mathrm{CH}_{2}$ ), 4.21 (s, 2H, $\left.\mathrm{CH}_{2}\right), 7.15-7.90$ (m, 13H, $\mathrm{ArH}+$ bezylic proton), $8.32(\mathrm{~s}, 1 \mathrm{H}, \mathrm{NH}$, exchangeable with $\left.\mathrm{D}_{2} \mathrm{O}\right) ;{ }^{13} \mathrm{C}-\mathrm{NMR}\left(\mathrm{CDCl}_{3}\right) \delta=12.75\left(\mathrm{CH}_{3}\right), 41.25,49.55,52.15,55.85$ 
$\left(4 \mathrm{CH}_{2}\right), 127.20,134.50(\mathrm{C}=\mathrm{C}), 106.25,126.10,152.35,161.42,161.85$ (PyridineC), $116.75(\mathrm{CN}), 116.50,125.30,136.15,155.25,126.23,127.80,128.45,129.25,134.55$, $136.15(\mathrm{Ph}-\mathrm{C}), 167.85(\mathrm{C}=\mathrm{O})$; MS m/z (\%): $603\left[\mathrm{M}^{+}\right](100)$ base peak corresponding to the molecular formula $\mathrm{C}_{32} \mathrm{H}_{25} \mathrm{Cl}_{2} \mathrm{FN}_{4} \mathrm{SO}$. Analysis for $\mathrm{C}_{32} \mathrm{H}_{25} \mathrm{Cl}_{2} \mathrm{FN}_{4} \mathrm{SO}$ (603.54): Calc. C, 63.68; H, 4.18; Cl, 11.75; N, 9.28; S, 5.31, Found C, 63.72; H, 4.22; Cl, 11.80; N, 9.32; S, 5.35

(8E)-3-Amino-4-(4-Chlorophenyl)-8-(4-chlorobenzylidene)-6-ethyl-N-aryl5,6,7,8-tetrahydrothieno[2,3-b][1,6]naphthyridine-2-carboxamides $(7 a, b)$

Method A:

A mixture of compound $\mathbf{6 a , b}(0.001$ mole) and 2-chloro- $N$-arylacetamide namely, 2-chloro$N$-( $p$-tolyl)acetamide or 2-chloro- $N$-(4-fluorophenyl)acetamide $(0.001$ mole $)$ was suspended in sodium ethoxide solution (10 $\mathrm{mg}$ sodium metal in $50 \mathrm{ml}$ ethanol) and refluxed for $2 \mathrm{~h}$. On cooling, the product precipitated was filtered off and recrystallized to afford 7 a,b respectively.

Method B:

A mixture of compound $3 \mathrm{~b}(0.45 \mathrm{~g}, 0.001 \mathrm{~mole})$ and 2-chloro- $N$-arylacetamide namely, 2chloro- $N$-( $p$-tolyl)acetamide or 2-chloro- $N$-(4-fluorophenyl)acetamide $(0.001$ mole) in sodium ethoxide solution (10 mg sodium metal in $50 \mathrm{ml}$ ethanol) was refluxed for $5 \mathrm{~h}$. The reaction mixture was cooled, poured onto cold water and the solid formed was filtered off and recrystallized to afford $\mathbf{7 a , b}$ respectively.

(8E)-3-Amino-4-(4-chlorophenyl)-8-(4-chlorobenzylidene)-6-ethyl-N-(4-methylphenyl)5,6,7,8-tetrahydrothieno[2,3-b][1,6]naphthyridine-2-carboxamide (7a)

Yield (80\%) [A], (45\%) [B], M.p. $172{ }^{\circ} \mathrm{C}$ (Ethanol), IR $(\mathrm{KBr}) \mathrm{v}=3483,3399\left(\mathrm{NH}_{2}\right)$, and 1645 (CO) $\mathrm{cm}^{-1}$; ${ }^{1} \mathrm{H}-\mathrm{NMR}\left(\mathrm{CDCl}_{3}\right) \delta: 0.98\left(\mathrm{t}, 3 \mathrm{H}, \mathrm{CH}_{3}\right), 2.24\left(\mathrm{~s}, 3 \mathrm{H}, \mathrm{CH}_{3}\right), 2.35\left(\mathrm{q}, 2 \mathrm{H}, \mathrm{CH}_{2}\right), 3.35$, $3.82\left(2 \mathrm{~d}, 4 \mathrm{H}, 2 \mathrm{CH}_{2}\right), 5.67\left(\mathrm{~s}, 2 \mathrm{H}, \mathrm{NH}_{2}\right.$, exchangeable with $\left.\mathrm{D}_{2} \mathrm{O}\right) ; 7.10-7.80(\mathrm{~m}, 13 \mathrm{H}, \mathrm{ArH}+$ benzylic proton), $8.13\left(\mathrm{~s}, 1 \mathrm{H}, \mathrm{NH}\right.$, exchangeable with $\left.\mathrm{D}_{2} \mathrm{O}\right) ;{ }^{13} \mathrm{C}-\mathrm{NMR}\left(\mathrm{CDCl}_{3}\right) \delta=12.65$, $20.66\left(2 \mathrm{CH}_{3}\right), 51.31,53.55,59.90\left(3 \mathrm{CH}_{2}\right), 128.25,137.65(\mathrm{C}=\mathrm{C}), 119.15,124.10,146.50$, 155.62, 156.77 (Pyridine-C), 128.75, 129.65, 129.77, 131.05, 132.35, 132.75, 133.55, 134.05, 135.76, 163.80 (Ph-C, Thiophene-C), 169.25 (C=O); MS m/z (\%): 599 [M $\mathrm{M}^{+}$(30) corresponding to the molecular formula $\mathrm{C}_{33} \mathrm{H}_{28} \mathrm{Cl}_{2} \mathrm{~N}_{4} \mathrm{SO}, 570$ (25), 509 base peak (100). Analysis for $\mathrm{C}_{33} \mathrm{H}_{28} \mathrm{Cl}_{2} \mathrm{~N}_{4} \mathrm{SO}$ (599.58): Calc. C, 66.11; $\mathrm{H}, 4.71 ; \mathrm{Cl}, 11.83 ; \mathrm{N}, 9.34 ; \mathrm{S}, 5.35$, Found $\mathrm{C}, 66.15 ; \mathrm{H}, 4.74 ; \mathrm{Cl}, 11.86 ; \mathrm{N}, 9.37$;, 5.31.

(8E)-3-Amino-4-(4-chlorophenyl)-8-(4-chlorobenzylidene)-6-ethyl-N-(4-fluorophenyl)5,6,7,8-tetrahydrothieno[2,3-b][1,6]naphthyridine-2-carboxamide (7b)

Yield (75\%) [A], (50\%) [B], M.p. $152{ }^{\circ} \mathrm{C}$ (Ethanol), IR $(\mathrm{KBr}) \vee=3450,3366\left(\mathrm{NH}_{2}\right)$, and 1655 (CO) $\mathrm{cm}^{-1} .{ }^{1} \mathrm{H}-\mathrm{NMR}\left(\mathrm{CDCl}_{3}\right) \delta$ : $\left.1.05\left(\mathrm{t}, 3 \mathrm{H}, \mathrm{CH}_{3}\right), 2.55\left(\mathrm{q}, 2 \mathrm{H}, \mathrm{CH}_{2}\right),\right), 3.40,3.70(2 \mathrm{~d}, 4 \mathrm{H}$, $\left.2 \mathrm{CH}_{2}\right), 5.67\left(\mathrm{~s}, 2 \mathrm{H}, \mathrm{NH}_{2}\right.$, exchangeable with $\left.\mathrm{D}_{2} \mathrm{O}\right) ; 7.10-7.80(\mathrm{~m}, 13 \mathrm{H}, \mathrm{ArH}+$ benzylic proton), $8.18\left(\mathrm{~s}, 1 \mathrm{H}, \mathrm{NH}\right.$, exchangeable with $\left.\mathrm{D}_{2} \mathrm{O}\right) ; \mathrm{MS} \mathrm{m} / \mathrm{z}(\%): 602\left[\mathrm{M}^{+}\right]$(30) corresponding to the molecular formula $\mathrm{C}_{32} \mathrm{H}_{25} \mathrm{Cl}_{2} \mathrm{FN}_{4} \mathrm{SO}, 442$ (25), 111 base peak (100). Analysis for $\mathrm{C}_{32} \mathrm{H}_{25} \mathrm{Cl}_{2} \mathrm{FN}_{4} \mathrm{SO}$ (603.54): Calc. $\mathrm{C}, 63.68 ; \mathrm{H}, 4.18 ; \mathrm{Cl}, 11.75 ; \mathrm{N}, 9.28 ; \mathrm{S}, 5.31$, Found C, 63.73; H, 4.22; Cl, 11.71; N, 9.32; S, 5.35. 
(7E)-11-(4-Chlorophenyl)-7-(4-chlorobenzylidene)-9-ethyl-2,3-diphenyl7,8,9,10-tetrahydropyrimido [4',5':4,5]thieno[2,3-b][1,6]naphthyridin-4(3H)-ones (8a,b)

A mixture of compound $7 \mathbf{a}, \mathbf{b}(0.001 \mathrm{~mole})$ and excess benzoyl chloride $(6 \mathrm{ml})$ was refluxed for $2 \mathrm{~h}$. Excess of benzoyl chloride was extracted several times with pet. ether (60-80) and the residue was recrystallized to give $\mathbf{8} \mathbf{a}, \mathbf{b}$.

(7E)-11-(4-Chlorophenyl)-7-(4-chlorobenzylidene)-9-ethyl-3-(4-methylphenyl)2-phenyl-7,8,9,10-tetrahydropyrimido[4',5':4,5]thieno[2,3-b][1,6]naphthyridin4(3H)-one (8a)

Yield (65\%), M.p. $205^{\circ} \mathrm{C}$ (Ethanol), IR (KBr) v = $1648(\mathrm{CO}) \mathrm{cm}^{-1} .{ }^{1} \mathrm{H}-\mathrm{NMR}\left(\mathrm{CDCl}_{3}\right) \delta: 1.05$ (t, 3H, $\left.\mathrm{CH}_{3}\right), 2.13\left(\mathrm{~s}, 3 \mathrm{H}, \mathrm{CH}_{3}\right), 2.45\left(\mathrm{q}, 2 \mathrm{H}, \mathrm{CH}_{2}\right), 3.65-3.85\left(\mathrm{~m}, 4 \mathrm{H}, 2 \mathrm{CH}_{2}\right), 6.95-7.77(\mathrm{~m}$, $18 \mathrm{H}, \mathrm{ArH}+$ benzylic proton), MS m/z (\%): $685\left[\mathrm{M}^{+}\right]$(44) corresponding to the molecular formula $\mathrm{C}_{40} \mathrm{H}_{30} \mathrm{Cl}_{2} \mathrm{~N}_{4} \mathrm{SO}, 559$ (35). Analysis for $\mathrm{C}_{40} \mathrm{H}_{30} \mathrm{Cl}_{2} \mathrm{~N}_{4} \mathrm{SO}$ (685.67): Calc. C, 70.07; $\mathrm{H}$, $4.41 ; \mathrm{Cl}, 10.34 ; \mathrm{N}, 8.17$; S, 4.68, Found C, 70.13; H, 4.45; Cl, 10.38; N, 8.23; S, 4.62.

(7E)-11-(4-Chlorophenyl)-7-(4-chlorobenzylidene)-9-ethyl-3-(4-fluorophenyl)2-phenyl-7,8,9,10-tetrahydropyrimido[4',5':4,5]thieno[2,3-b][1,6]naphthyridin4(3H)-one (8b)

Yield (60\%), M.p. $192{ }^{\circ} \mathrm{C}$ (Ethanol), IR (KBr) v = $1645(\mathrm{CO}) \mathrm{cm}^{-1} .{ }^{1} \mathrm{H}-\mathrm{NMR}\left(\mathrm{CDCl}_{3}\right) \delta: 1.08$ $\left(\mathrm{t}, 3 \mathrm{H}, \mathrm{CH}_{3}\right), 2.35\left(\mathrm{q}, 2 \mathrm{H}, \mathrm{CH}_{2}\right), 3.53-3.77\left(\mathrm{~m}, 4 \mathrm{H}, 2 \mathrm{CH}_{2}\right), 7.10-7.85(\mathrm{~m}, 18 \mathrm{H}, \mathrm{ArH}+$ benzylic proton); ${ }^{13} \mathrm{C}-\mathrm{NMR}\left(\mathrm{CDCl}_{3}\right) \delta=12.55\left(\mathrm{CH}_{3}\right), 53.50,56.35,60.40\left(3 \mathrm{CH}_{2}\right), 127.60$, $136.50(\mathrm{C}=\mathrm{C}), 117.25,124.30,126.23,127.80,129.45,133.50,135.35,137.20(\mathrm{Ph}-\mathrm{C})$, 148.55, 156.32, 158,53 (Pyridine-C), 147.35, 162,10, 165.34 (Pyrimidine-C),167.25 (C=O); MS m/z (\%): $689\left[\mathrm{M}^{+}\right]$(44) corresponding to the molecular formula $\mathrm{C}_{39} \mathrm{H}_{27} \mathrm{Cl}_{2} \mathrm{FN}_{4} \mathrm{SO}, 559$ (35). Analysis for $\mathrm{C}_{39} \mathrm{H}_{27} \mathrm{Cl}_{2} \mathrm{FN}_{4} \mathrm{SO}$ (689.63): Calc. C, 67.92; $\mathrm{H}, 3.95 ; \mathrm{Cl}, 10.28 ; \mathrm{N}, 8.12 ; \mathrm{S}$, 4.65, Found C, 67.96; H, 4.41; Cl, 10.22; N, 8.27; S, 4.65.

(7E)-11-(4-Chlorophenyl)-7-(4-chlorobenzylidene)-9-ethyl-3-phenyl-7,8,9,10tetrahydro[1,2,3]triazino [4',5':4,5]thieno[2,3-b][1,6]naphthyridin-4(3H)-ones $(9 a, b)$

To a mixture of compound $7 \mathbf{a}, \mathbf{b}(0.001$ mole $)$ in conc. hydrochloric acid $(5 \mathrm{ml})$ and glacial

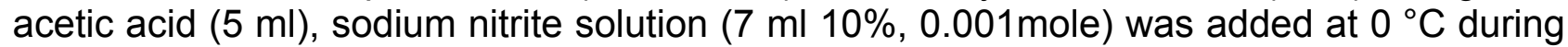
5 minutes with stirring. After half hour, the reaction mixture was poured onto water, the solid thus precipitated was collected, dried and recrystallized to give $\mathbf{9 a , b}$.

(7E)-11-(4-Chlorophenyl)-7-(4-chlorobenzylidene)-9-ethyl-3-(4-methylphenyl)7,8,9,10-tetrahydro[1,2,3]triazino [4',5':4,5]thieno[2,3-b][1,6]naphthyridin-4(3H)-one (9a)

Yield (85\%), M.p. $216{ }^{\circ} \mathrm{C}$ (Ethanol), IR $(\mathrm{KBr})=\mathrm{v} 1668(\mathrm{CO}) \mathrm{cm}^{-1} .{ }^{1} \mathrm{H}-\mathrm{NMR}\left(\mathrm{CDCl}_{3}\right) \delta: 1.07$ (t, 3H, $\left.\mathrm{CH}_{3}\right), 2.42$ (s, 3H, $\left.\mathrm{CH}_{3}\right), 3.01$ (q, 2H, $\left.\mathrm{CH}_{2}\right), 3.75,4.12\left(2 \mathrm{~d}, 4 \mathrm{H}, 2 \mathrm{CH}_{2}\right), 7.20-7.85(\mathrm{~m}$, $13 \mathrm{H}, \mathrm{ArH}+$ benzylic proton); ${ }^{13} \mathrm{C}-\mathrm{NMR}\left(\mathrm{CDCl}_{3}\right) \delta=13.25,22.35\left(2 \mathrm{CH}_{3}\right), 49.20,52.95$, $57.70\left(3 \mathrm{CH}_{2}\right), 129.80,138.30$ (C=C), 122.15, 128.45, 127.80, 129.40, 133.50, 134.65, 135.35, 138.40 (Ph-C), 147.55, 155.45, 157, 25 (Pyridine-C), 139.15, 152,35 (PyrimidineC), $168.65(\mathrm{C}=0)$; MS m/z (\%): $609\left[\mathrm{M}^{+}\right]$base peak (100) corresponding to the molecular formula $\mathrm{C}_{33} \mathrm{H}_{25} \mathrm{Cl}_{2} \mathrm{~N}_{5} \mathrm{SO}, 580$ (75). Analysis for $\mathrm{C}_{33} \mathrm{H}_{25} \mathrm{Cl}_{2} \mathrm{~N}_{5} \mathrm{SO}$ (609.11) Calc. C, 65.01; $\mathrm{H}$, 4.14; Cl, 11.48; N, 11.49; S, 5.25, Found C, 65.06; H, 4.18; Cl, 11.53; N, 11.52; S, 5.28. 
(7E)-11-(4-Chlorophenyl)-7-(4-chlorobenzylidene)-9-ethyl-3-(4-fluorophenyl)-7,8,9,10tetrahydro[1,2,3]triazino $\left[4^{\prime}, 5^{\prime}: 4,5\right]$ thieno[2,3-b][1,6]naphthyridin-4(3H)-one (9b)

Yield (80\%), M.p. $227^{\circ} \mathrm{C}$ (Ethanol), IR (KBr) v = $1655(\mathrm{CO}) \mathrm{cm}^{-1} .{ }^{1} \mathrm{H}-\mathrm{NMR}\left(\mathrm{CDCl}_{3}\right) \delta: 1.25$ $\left(\mathrm{t}, 3 \mathrm{H}, \mathrm{CH}_{3}\right), 3.01\left(\mathrm{q}, 2 \mathrm{H}, \mathrm{CH}_{2}\right)$, ), 3.88, $4.25\left(2 \mathrm{~d}, 4 \mathrm{H}, 2 \mathrm{CH}_{2}\right), 7.20-7.85(\mathrm{~m}, 13 \mathrm{H}, \mathrm{ArH}+$ benzylic proton), MS m/z (\%): $613\left[\mathrm{M}^{+}\right]$(35) corresponding to the molecular formula $\mathrm{C}_{32} \mathrm{H}_{22} \mathrm{Cl}_{2} \mathrm{FN}_{5} \mathrm{SO}, 585$ base peak (100), 458 (85). Analysis for $\mathrm{C}_{32} \mathrm{H}_{22} \mathrm{Cl}_{2} \mathrm{FN}_{5} \mathrm{SO}$ (613.11): Calc. C, 62.63; H, 4.18; Cl, 11.41 N, 11.42; S, 5.21, Found C, 62.67; H, 4.23; Cl, 11.46 N, 11.45; S, 5.26 .

\section{$\mathrm{N}-\{(8 \mathrm{E})-4-(4-C h l o r o p h e n y l)-8-(4-c h l o r o b e n z y l i d e n e)-3-c y a n o-6-e t h y l-$} 5,6,7,8-tetrahydro-4H-pyrano[3,2-c]pyridin-2-yl\}acetamide (10)

A mixture of compound $2(0.01$ mole $)$ in acetic anhydride $(10 \mathrm{ml})$ was refluxed for $1 \mathrm{~h}$. After cooling the reaction mixture was poured onto cold water, the solid formed was filtered off, dried and recrystallized to give 10. Yield (65\%), M.p. $205{ }^{\circ} \mathrm{C}$ (Ethanol), IR $(\mathrm{KBr}) \mathrm{v}=2195$

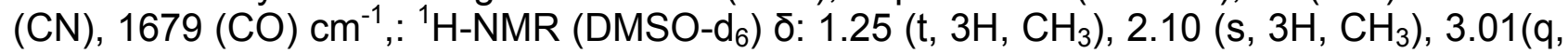
$\left.2 \mathrm{H}, \mathrm{CH}_{2}\right)$, ), 3.27, $3.60\left(2 \mathrm{~d}, 4 \mathrm{H}, 2 \mathrm{CH}_{2}\right), 3.95$ (s, 1H, pyran proton), 7.10-7.85 (m, $9 \mathrm{H}, \mathrm{ArH}+$ benzylic proton) and $8.24\left(\mathrm{~s}, 1 \mathrm{H}, \mathrm{NH}\right.$, exchangeable with $\left.\mathrm{D}_{2} \mathrm{O}\right), \mathrm{MS} \mathrm{m} / \mathrm{z}(\%): 479\left[\mathrm{M}^{+}\right](22)$ corresponding to the molecular formula $\mathrm{C}_{26} \mathrm{H}_{23} \mathrm{Cl}_{2} \mathrm{~N}_{3} \mathrm{O}_{2}, 436$ base peak (100), 393 (55). Analysis for $\mathrm{C}_{26} \mathrm{H}_{23} \mathrm{Cl}_{2} \mathrm{~N}_{3} \mathrm{O}_{2}$ (479.11): Calc. C, 65.12; $\mathrm{H}, 4.84 ; \mathrm{Cl}, 14.60 ; \mathrm{N}, 8.77$, Found $\mathrm{C}$, $65.17 ; \mathrm{H}, 4.80 ; \mathrm{Cl}, 14.65 ; \mathrm{N}, 8.71$.

\section{(9E)-5-(4-Chlorophenyl)-9-(4-chlorobenzylidene)-7-ethyl-3,5,6,7,8,9-hexahydro- 4H-pyrido[3',4':5,6]pyrano[2,3-d]pyrimidin-4-one (11)}

A mixture of compound 2 (0.01 mole) in formic acid $(10 \mathrm{ml})$ was refluxed for $1 \mathrm{hr}$. After cooling the reaction mixture was poured onto cold water, the solid formed was filtered off, dried and recrystallized to give 11 Yield (75\%), M.p. $183^{\circ} \mathrm{C}$ (Ethanol), IR $(\mathrm{KBr}) \mathrm{v}=1669$ (CO) $\mathrm{cm}^{-1},{ }^{1} \mathrm{H}-\mathrm{NMR}\left(\mathrm{DMSO}_{-} \mathrm{d}_{6}\right) \delta$ : $1.25\left(\mathrm{t}, 3 \mathrm{H}, \mathrm{CH}_{3}\right), 3.01$ (q, 2H, $\left.\mathrm{CH}_{2}\right)$, ), 3.35, $3.60(2 \mathrm{~d}$, $\left.4 \mathrm{H}, 2 \mathrm{CH}_{2}\right), 4.10(\mathrm{~s}, 1 \mathrm{H}$, pyran proton) 7.20-7.65 (m, 9H, ArH + benzylic proton), 7.95 (s, $1 \mathrm{H}$, pyrimidine proton), $8.25\left(\mathrm{~s}, 1 \mathrm{H}, \mathrm{NH}\right.$, exchangeable with $\left.\mathrm{D}_{2} \mathrm{O}\right), \mathrm{MS} \mathrm{m} / \mathrm{z}(\%): 465\left[\mathrm{M}^{+}\right]$ (50) corresponding to the molecular formula $\mathrm{C}_{25} \mathrm{H}_{21} \mathrm{Cl}_{2} \mathrm{~N}_{3} \mathrm{O}_{2}, 436$ base peak (100), 393 (55). Analysis for $\mathrm{C}_{25} \mathrm{H}_{21} \mathrm{Cl}_{2} \mathrm{~N}_{3} \mathrm{O}_{2}$ (465.10): Calc. C, 64.50; $\mathrm{H}, 4.55 ; \mathrm{Cl}, 15.04 ; \mathrm{N}, 9.03$, Found $\mathrm{C}, 64.55 ; \mathrm{H}, 4.51 ; \mathrm{Cl}, 15.09 ; \mathrm{N}, 9.07$.

\section{Ethyl \{(8E)-4-(4-chlorophenyl)-8-(4-chlorobenzylidene)-3-cyano-6-ethyl- 5,6,7,8-tetrahydro-4H-pyrano[3,2-c]pyridin-2-yl\}imidoformate (12)}

A mixture of compound 2 (0.01 mole), triethyl ortho-formate (0.01 mole) and acetic anhydride $(20 \mathrm{ml})$ was refluxed for $6 \mathrm{~h}$. The solvent was removed under vacuum,, the residue obtained was solidified by ether and recrystallized to give 12. Yield (75\%), M.p. $155^{\circ} \mathrm{C}$ (Ethanol), IR (KBr) v = $2980(\mathrm{CH}), 2218(\mathrm{CN}), 1630(\mathrm{C}=\mathrm{N}) \mathrm{cm}^{-1},{ }^{1} \mathrm{H}-\mathrm{NMR}$ (DMSO$\left.\mathrm{d}_{6}\right) \delta: 1.10,1.25\left(2 \mathrm{t}, 6 \mathrm{H}, 2 \mathrm{CH}_{3}\right), 3.01\left(\mathrm{q}, 2 \mathrm{H}, \mathrm{CH}_{2}\right)$, ), 3.57, $3.80(2 \mathrm{~d}, 4 \mathrm{H}),, 3.95\left(\mathrm{q}, 2 \mathrm{H}, \mathrm{CH}_{2}\right)$, $4.15(\mathrm{~s}, 1 \mathrm{H}$, pyran proton), 7.10-7.85 (m, 10H, ArH + benzylic proton+CH); MS m/z (\%): $492\left[\mathrm{M}^{+}\right]$(85) corresponding to the molecular formula $\mathrm{C}_{27} \mathrm{H}_{25} \mathrm{Cl}_{2} \mathrm{~N}_{3} \mathrm{O}_{2}, 393$ (100) base peak. Analysis for $\mathrm{C}_{27} \mathrm{H}_{25} \mathrm{Cl}_{2} \mathrm{~N}_{3} \mathrm{O}_{2}$ (393.13): Calc. C, 65.70; $\mathrm{H}, 5.11 ; \mathrm{Cl}, 14.18 ; \mathrm{N}, 8.52$, Found $\mathrm{C}, 65.75 ; \mathrm{H}, 5.14 ; \mathrm{Cl}, 14.22 ; \mathrm{N}, 8.56$ 
(9E)-5-(4-Chlorophenyl)-9-(4-chlorobenzylidene)-7-ethyl-4-imino-6,7,8,9-tetrahydro4H-pyrido[3',4':5,6]pyrano[2,3-d]pyrimidin-3(5H)-amine (13)

A mixture of compound 12 (0.01 mole) and hydrazine hydrate $(99 \%, 5 \mathrm{ml})$ in ethanol $(50 \mathrm{ml})$ was stirred at room temperature for $1 \mathrm{~h}$. the solid obtained was filtered off and recrystallized to give 13. Yield $(55 \%)$, M.p. $179{ }^{\circ} \mathrm{C}(\mathrm{EtOH}), \mathrm{IR}(\mathrm{KBr}) \mathrm{v}=3316,3335\left(\mathrm{NH}_{2}\right)$, $1635(\mathrm{C}=\mathrm{N}) \mathrm{cm}^{-1},{ }^{1} \mathrm{H}-\mathrm{NMR}\left(\mathrm{DMSO}-\mathrm{d}_{6}\right) \delta$ : $0.97\left(\mathrm{t}, 3 \mathrm{H}, \mathrm{CH}_{3}\right), 2.72\left(\mathrm{q}, 2 \mathrm{H}, \mathrm{CH}_{2}\right.$ ), 3.75-4.18 (m, $\left.4 \mathrm{H}, 2 \mathrm{CH}_{2}\right), 4.26$ (s, 1H, pyran proton), $5.65\left(\mathrm{~s}, 2 \mathrm{H}, \mathrm{NH}_{2}\right.$, exchangeable by $\left.\mathrm{D}_{2} \mathrm{O}\right), 7.30$ $7.95(\mathrm{~m}, 9 \mathrm{H}, \mathrm{ArH}+$ benzylic proton) and 8.15 (s, 1H, pyrimidine proton), MS m/z (\%): 479 $\left[\mathrm{M}^{+}\right]$(83) corresponding to the molecular formula $\mathrm{C}_{25} \mathrm{H}_{23} \mathrm{Cl}_{2} \mathrm{~N}_{5} \mathrm{O}, 463$ base peak (100), 436 (90). Analysis for $\mathrm{C}_{25} \mathrm{H}_{23} \mathrm{Cl}_{2} \mathrm{~N}_{5} \mathrm{O}$ (479.13): Calc. C, 62.61; $\mathrm{H}, 4.84 ; \mathrm{Cl}, 14.60 ; \mathrm{N}, 14.61$, Found C, 62.66; H, 4.80; Cl, 14.64; N, 14.65.

\section{Pharmacological Assay}

\section{Anti-arrhythmic Activity [19-24].}

\section{Purpose and Rational}

The plant alkaloid aconitine persistently activates sodium channel. Infusion of aconitine in the anesthetized rat causes ventricular arrhythmias. Drugs considered to have antiarrhythmic properties can be tested in aconitine-intoxicated rats.

\section{Procedure}

Male Ivanovas rats weighing $300-350 \mathrm{~g}$ are used. The animals are anesthetized by intra peritoneal injection of $1.25 \mathrm{~g} / \mathrm{kg}$ urethane: $5 \mathrm{mg} / \mathrm{kg}$ aconitine dissolved in $0.1 \mathrm{~N} \mathrm{HNO}_{3}$ is adminstered by continuous infusion into the saphenous vein of $0.1 \mathrm{ml} / \mathrm{min}$ and the ECG in lead II is recorded every 30 seconds. The test compound is injected IV at a screening dose of $3 \mathrm{mg} / \mathrm{kg} 5 \mathrm{~min}$ before the start of the aconitine infusion, 24 animals are used per compound.

Animals were obtained from the animal house colony of the National Research Center, Cairo, Egypt. The animals were allowed free access to water and were kept on a constant standard diet. All animal experiments were carried out in compliance with the institutional guidelines on the use of living animals.

\section{Evaluation}

The anti-arrhythmic effect of a test compound is measured by the amount of aconitine $/ 100 \mathrm{~g}$ animal.

(Duration of infusion) which induces:

Ventricular extra systoles.

Ventricular tachycardia.

Ventricular fibrillation.

Higher doses of aconitine in the treated group as compared to an untreated control group are an indication of anti-arrhythmic activity. Statistical significance between the groups is assessed by the student's T-test. 


\section{Authors' Statements}

\section{Competing Interests}

The authors declare no conflict of interest.

\section{Animal Rights}

The institutional and (inter)national guide for the care and use of laboratory animals was followed. See the experimental part for details.

\section{References}

[1] Amr AE, Mohamed AM, Mohamed SF, Abdel-Hafez NA, Hammam AG.

Anticancer activities of some newly synthesized pyridine, pyrane, and pyrimidine derivatives.

Bioorg Med Chem. 2006; 14: 5481-5488.

doi:10.1016/j.bmc.2006.04.045

[2] Hammam AG, Abdel-Salam OI, Mohamed AM, Abdel-Hafez NA.

Novel flouro substituted benzo[b]pyran with anti-lung cancer activity.

Ind J Chem. 2005; 44B: 1887-1893.

[3] Hammam AG, Fahmy AFM, Amr AE, Mohamed AM.

Synthesis of novel tricyclicheterocyclic compounds as potential anticancer agents using chromanone and thiochromanoneas synthons.

Ind J Chem. 2003; 42B: 1985-1993.

[4] Hammam AG, Sharaf MA, Abdel-Hafez NA.

Synthesis and anti-cancer activity of pyridineand thiazolopyrimidine derivatives using

1-ethylpiperidone as a synthon.

Ind J Chem. 2001; 40B: 213-221.

[5] Hammam AG, Abdel-Hafez NA, Midura WH, Mikolajczyk M.

Chemistry of Seven-Membered Heterocycles, VI. Synthesis of Novel Bicyclic Heterocyclic Compounds as Potential Anticancer and Anti-HIV Agents.

Z Naturforsch. 2000; 55B: 417-424.

[6] DeClercq E.

Potential of bromovinyldeoxyuridine in anticancer chemotherapy.

Anticancer Res. 1986; 6: 549-556.

PMid:3752935

[7] DeClercq E.

Chemotherapeutic approaches to the treatment of the acquired immune deficiency syndrome (AIDS).

J Med Chem. 1986; 29: 1561-1569.

doi:10.1021/jm00159a001

[8] Munchhof MJ, Beebe JS, Casavant JM, Cooper BA, Doty JL, Higdon RC, Hillerman SM, Soderstrom Cl, Knauth EA, Marx MA, Rossi AM, Sobolov SB, Sun J.

Design and SAR of thienopyrimidine and thienopyridine inhibitors of VEGFR-2 kinase activity.

Bioorg Med Chem Lett. 2004; 14: 21-24.

doi:10.1016/j.bmcl.2003.10.030

[9] Abdel-Fattah B, Kandeel MM, Abdel-Hakeem M, Fahmy ZM.

Synthesis of Certain Fused Thienopyrimidines of Biological Interest.

J Chin Chem Soc. 2006; 53: 403-412.

[10] Nehad AA, Amr AE, Alhusain Al.

Synthesis, Reactions, and Pharmacological Screening of Heterocyclic Derivatives Using Nicotinic Acid as a Natural Synthon.

Monatsh Chem. 2007; 138: 559-567.

doi:10.1007/s00706-007-0628-z 
[11] Amr AE, Abdel-Latif NA, Abdalla MM.

Synthesis and antiandrogenic activity of some new 3-substituted androstano[17,16-c]-5'-arylpyrazoline and their derivatives.

Bioorg Med Chem. 2006; 14: 373-384.

doi:10.1016/j.bmc.2005.08.024

[12] Amr AE, Sayed HH, Abdulla MM.

Synthesis and Reactions of Some New Substituted Pyridine and Pyrimidine Derivatives as Analgesic, Anticonvulsant and Antiparkinsonian Agents.

Arch Pharm. 2005; 338: 433-440.

doi:10.1002/ardp.200500982

[13] Amr AE.

Synthesis of some new linear and chiral macrocyclic pyridine carbazides as analgesic and anticonvulsant agents:

Z Naturforsch. 2005; 60B, 990-998.

[14] Amr AE, Abou-Galia MH, Abdulla MM.

Synthesis of novel macrocyclic peptide-calix[4]arenas and peptide-pyridines as precursors for potential molecular metallacages, chemosensors and biologically active candidates.

Z Naturforsch: 2006; 61B: 1335-1345.

[15] Amr AE, Abdulla MM.

Synthesis and Anti-inflammatory Activities of New Cyanopyrane Derivatives Fused with Steroidal Nuclei.

Arch Pharm. 2006; 339: 88-95.

doi:10.1002/ardp.200500209

[16] Atherall JF, Hough TL, Linjell SA, Mahony MJ, Anne ES.

US Patent 2003; 6,541, 630B.

[17] Wang YD, Johnson S, Powell D, McGinnis JP, Miranda M; Rabindran SK:

Inhibition of tumor cell proliferation by thieno[2,3-d]pyrimidin-4(1H)-one-based analogs.

Bioorg Med Chem Lett. 2005; 15: 3763-3766:

doi:10.1016/j.bmcl.2005.05.127

[18] Jenning LD, Kincaid SL, Wang YD, Krishnamurthy G, Beyer CF,. McGinnis JP, Miranda M, Discafani CM, Rabindran SK.

Parallel synthesis and biological evaluation of 5,6,7,8-tetrahydrobenzothieno-[2,3-d]pyrimidin-4(3H)one cytotoxic agents selective for p21-deficient cells.

Bioorg Med Chem Lett. 2005; 15: 4731-4735.

doi:10.1016/j.bmcl.2005.07.072

[19] Walker MJA, Curtius MJ, Hearse DJ, Campbell RWF, Jams MJ, Yellon DM, Coker SM, Harness JB, Harron DWG, Miggins AJ, Julian DG, Lab MJ, Manning AS, Northover BJ, Parratt JR, Riemrsma RA, Riva E, Russell DC, Sheridan DJ, Winslow E, Woodward B.

The Lambeth Conventions: guidelines for the study of arrhythmias in ischaemia, infarction, and reperfusion.

Cardiovasc Res. 1988; 22: 447-455.

doi:10.1093/cvr/22.7.447

[20] Vaille AM, Tella SD, Maldonado J, Vanelle P.

Selectivity of a $\mathrm{CaCl} 2$ continuous infusion screening method in rats.

Meth Find Exp Clin Pharmacol. 1992; 14: 183-187.

PMid:1625502

[21] Bazzani C, Genedani S, Tugliavini S, Bertolini A.

Putrescine reverses aconitine-induced arrhythmia in rats.

J Pharm Pharmacol. 1989; 41: 651-653:

PMid:2573715 
[22] De Clerk F, Rong LH.

R.56 865, a Na+/Ca2+-Overload inhibitor, protects against aconitine-induced cardiac arrhythmias in Vitro:

J Cardiovasc Pharmacol. 1993; 22: 120-125.

PMid:7690083

[23] Winslow E.

Evaluation of antagonism of aconitine-induced dysrhythmias in mice as a method of detecting and assessing antidysrhythmic activity.

Br J Pharmacol. 1980; 71: 615-622.

PMid:7470765

[24] Winslow E.

Hemodynamic and Arrhythmogenic Effects of Aconitine Applied to the Left Atria of Anesthetized Cats. Effects of Amiodarone and Atropine.

J Cardiovasc Pharmac. 1981; 3: 87-100.

PMid:6160357 